\title{
Prolonged Delirium Secondary to Hypoxic-ischemic Encephalopathy Following Cardiac Arrest
}

\author{
Jegan Yogaratnam, Rajesh Jacob, Sandeep Naik, Harish Magadi, Kang Sim \\ Institute of Mental Health/Woodbridge Hospital, Singapore
}

Hypoxic-ischemic brain injury encompasses a complex constellation of pathophysiological and cellular brain injury induced by hypoxia, ischemia, cytotoxicity, or combinations of these mechanisms and can result in poor outcomes including significant changes in personality and cognitive impairments in memory, cognition, and attention. We report a case of a male patient with normal premorbid functioning who developed prolonged delirium following hypoxic-ischemic brain insults subsequent to cardiac arrest. The case highlights the importance of adopting a multidisciplinary treatment approach involving the coordinated care of medical and nursing teams to optimise management of patients suffering from such a debilitating organic brain syndrome.

KEY WORDS: Cardiac arrest; Neurologic manifestations; Sequelae; Delirium.

\section{INTRODUCTION}

Hypoxic ischemic encephalopathy (HIE) is a condition that occurs when the entire brain is deprived of an adequate oxygen supply, and is often a complication of cardiac arrest or profound hypotension, or consequent to suffocation, airway obstruction, drowning and chemical exposure such as carbon monoxide poisoning. ${ }^{1)}$ The clinical pattern and outcome depend on the severity of the initial insult, the effectiveness of immediate resuscitation and transfer to the intensive care unit of a medical unit, and the post-resuscitation management of the intensive care unit. ${ }^{1)}$ Overall, the prognosis can be poor and only a quarter of patients survive to be discharged from the hospital, and often with severe neurological or cognitive deficits including significant impairments in memory and attention. ${ }^{1,2)}$ In the context of sparse literature reports on prolonged delirium following cardiac arrest related HIE, we report a case of a male patient with normal premorbid functioning who developed prolonged confusional state following repeated hypoxic-ischemic insults to the brain subsequent to cardiac arrest and try to highlight the chal-

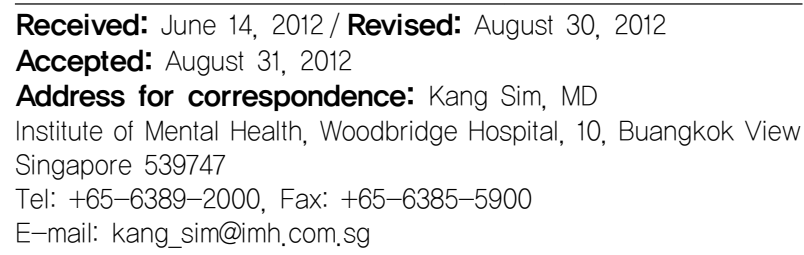

lenges encountered during his clinical course and management.

\section{CASE}

A 59-year-old male was transferred from a general hospital to the tertiary psychiatric hospital in August 2011 after treatment of heart failure. He had been "healthy" and functioning well premorbidly and had multiple medical conditions such as hypertension, diabetes mellitus and hyperlipidaemia. He was first admitted to the cardiology unit of a general hospital in January 2011 after he collapsed during jogging. Following basic cardiac life support he was rushed to the emergency room of the general hospital. He was diagnosed to have acute myocardial infarction secondary to triple vessel disease and was arranged for coronary artery bypass surgery when his physical condition was stabilised. The electroencephalography and computerised tomography (CT) scans of the brain done on the day of admission in view of his fall were normal but the magnetic resonance imaging of the brain 10 days later showed mild generalized involutional change and disproportionate atrophy of basal ganglia and hippocampus. He regained full consciousness about 5 hours after the admission and was noted to have resting tremors and mild cogwheel rigidity before the administration of any psychotropic medications. He showed mild impairment in both retrograde and anterograde memory, which was greater for short term than long term memory.

(a) This is an Open-Access article distributed under the terms of the Creative Commons Attribution Non-Commercial License (http://creativecommons.org/licenses/by-nc/3.0) which permits unrestricted non-commercial use, distribution, and reproduction in any medium, provided the original work is properly cited. 
Subsequently, he was observed to have fluctuating conscious state and agitation but no localised motor or sensory neurological deficits. Laboratory investigations, which were performed including full blood count, renal, liver, thyroid function tests, and infective screen did not reveal any abnormalities. He was then discharged to a unit for cognitive rehabilitation in April 2011 and he was receiving oral haloperidol $2.5 \mathrm{mg}$ daily for his agitation. For the next two months at the rehabilitation centre, he remained disoriented to time, place and person and can be emotionally labile at times.

In June 2011, he underwent his scheduled coronary artery bypass surgery. Intra-operatively he did not have any complications related to the surgical procedures or anaesthesia. However, postoperatively, he continued to be agitated and more disoriented to time, place and person, which persisted for the next 3 months. A month after his cardiac procedures, he was transferred to a residential facility for further cognitive rehabilitation and he was receiving oral haloperidol drops $3 \mathrm{mg}$ daily for his agitation. He was subsequently re-admitted twice to a general hospital after he punched a nursing home staff on both occasions. In order to control his agitation, aggression and perceptual abnormalities, many antipsychotic medications were tried. His psychotropic medication was finally changed to olanzapine $2.5 \mathrm{mg}$ daily as he exhibited extrapyramidal side effects attributable to the haloperidol and risperidone and orthostatic hypotension to quetiapine. The treating team also tried mood stabilisers but he developed adverse drug reactions such as maculopapular rash with lithium, sodium valproate and Steven Johnson syndrome with carbamazepine. As he also assaulted a staff nurse in the general hospital, he was transferred to a tertiary psychiatric hospital for further management of his neuropsychiatric condition.

At the psychiatric facility, his disorientation to time, place and person continued. He was agitated frequently, which needed rapid tranquilization and brief periods of minimal physical restraint for his safety. Formal cognitive assessment was attempted using Mini Mental State Examination (MMSE) and clock drawing test, but could not be completed owing to his uncooperative behaviour. The repeat CT scan of his brain revealed increased generalized volume loss of his brain. The nurses noted "sun downing effect" as he tended to become more disoriented and agitated towards the latter part of the day. He had impaired sleep wake cycle in that he was noted to be sleepy during the day time and more alert and disturbed at night. He also insisted on being only attended by female staff and even requested for alcohol in the ward although he was a teetotaller. As he continued to exhibit high risk of agitation, he was transferred to a high dependency psychiatric care unit for closer observation and management.

During his four weeks' stay in the high dependency psychiatric care unit, he continued to have cognitive deficits in orientation, attention and concentration, memory which mainly worsened at night. Gradually his agitation responded to sulpiride $800 \mathrm{mg}$ daily which was chosen due to the lower tendency for extra pyramidal symptoms. He could repeat sentences but was unable to recall the names of his close family members. Formal cognitive testing was attempted several times but to no avail due his uncooperativeness. Repeated laboratory investigations including full blood count, renal, liver and thyroid function tests, serum calcium and magnesium levels and cardiac enzymes were normal and his electrocardiogram showed sinus rhythm with left ventricular hypertrophy. Since his cognitive functions did not improve, he was referred to a psycho-geriatrician and subsequently transferred to the psychogeriatric ward for further management.

In the psychogeriatric ward, his clinical status remained the same for another few months. Nine months after the index cardiac event, the delirious features including disorientation to place and person, impaired attention and concentration, altered sensorium, perceptual disturbances such as visual and auditory hallucinations, emotional lability and psychomotor disturbances slowly improved. The altered sensorium, perceptual and psychomotor disturbances finally completely resolved with residual short term and long term memory deficits. He needed assistance for all his basic activities of daily living including toileting and feeding. He became more cooperative with the formal cognitive testing scoring 14 out 30 on MMSE. Input from the multi-disciplinary team involving psychiatric nurses,occupational therapist, physiotherapist, speech therapist and psychologist was sought to manage and improve his functional status. His social issues were addressed by the medical social worker. He was also reviewed by the visiting geriatrician on a regular basis for the management of his underlying medical conditions and he was prescribed clopidogrel $75 \mathrm{mg}$ daily, frusemide $20 \mathrm{mg}$ daily, perindopril $2 \mathrm{mg}$ daily, simvastatin $40 \mathrm{mg}$ daily and gliclazide $20 \mathrm{mg}$ daily for his medical conditions. Overall, he was diagnosed with dementia which was secondary to cardiac arrest related HIE and complicated by prolonged delirium-which started following the incipient episode of cardiac arrest in January 2011 with further exacerbation 
after the cardiac bypass surgery. The other possibility of depressive episode resulting in cognitive deficits such as "pseudo dementia" was ruled out, as there was no clinical evidence to suggest a depressive episode that preceded the cognitive disturbance in this patient.

At the time of writing this case report, the patient still remained confused with significant impairment in short term and long term memory, although there was no fluctuation in consciousness or perceptual abnormalities. He needs assistance in all his basic activities of daily living and he displays aggressive behaviour in the ward on and off.

\section{DISCUSSION}

This case is unique in the clinical presentation of prolonged delirium following hypoxic-ischemic insults to the brain subsequent to the cardiac arrest. Since the clinical picture in the initial 9 months clearly pointed towards delirium (fluctuating conscious level, perceptual and psychomotor abnormalities, impaired sleep wake cycle) without any preceding cognitive impairment, the diagnosis of dementia was made only after the resolution of delirium. The differentiation between dementia and delirium (especially if it is prolonged) may seem daunting which is understandable given the complexities of these conditions and the overlap in symptomatology. Delirium and dementia (especially in advanced stages) can display similar clinical symptoms such as sleep disturbance, impaired speech, perceptual disturbance, disturbed thought process, disorientation and memory disturbances creating difficulties in the diagnosis. Hypoxic-ischemic brain injury encompasses a complex constellation of pathophysiological and cellular insults to the brain induced by hypoxia, ischemia, cytotoxicity, or combination of these mechanisms. Clinical manifestations of patients with hypoxic-ischemic brain injury include changes in personality, behaviour, executive function deficits, deficits in spatial, verbal memory and recall and disorders of visuoperceptual function and expressive language, although language function is relatively spared. ${ }^{1)}$ In this patient, the language functions were significantly impaired. Hypoxicischemic encephalopathy accompanying cardiac arrest is a common cause of long-term neurological dysfunction. With the improvement in pre-hospital emergency systems, larger numbers of people are resuscitated from cardiac arrests, although with the increased prospect of neurological sequelae. Neurological impairment after cardiac arrest is dependent on the degree of brain damage suffered during the arrest. Although the duration and severity of brain ischemia is often difficult to determine, clinicians are often faced with difficult issues related to predicting outcome associated with awakening and long-term neurological deficits after the arrest. Neurological impairments of HIE following cardiac arrest can range from mild cognitive deficits to severe motor and cognitive deficits that preclude independence in many activities of daily living. Delirium is defined as a transient, usually reversible cause of cerebral dysfunction and manifests clinically with a wide range of neuropsychiatric abnormalities. The usual duration ranges from hours to days but delirium can last up to 6 months according to the International Classification of Diseases-10th edition (ICD-10). Of note, the delirium following HIE can occur in up to $28 \%$ of patients following a cardiac arrest and often only lasts a few hours or days ${ }^{3)}$ unlike the unusual protracted course as was manifested in this case.

The molecular mechanisms underlying HIE are complex and diverse. They include a neurobiological cascade of time-dependent alterations in neuronal function, metabolism and morphology and an increase in neuronal excitability with calcium influx leading to the formation of oxygen free radicals. ${ }^{4)}$ Neuropathological and structural brain imaging studies of patients with cerebral anoxia clearly demonstrate that a network of brain regions can be affected, including the watershed areas of the cortex, thalamus, the basal ganglia and the hippocampus with the latter two brain regions with high metabolic rates being particularly vulnerable to hypoxic-ischemic damage ${ }^{5)}$ which was observed in this patient. In the first few days, diffusion weighted imaging (DWI) and fluid attenuated inversion recovery (FLAIR) images of the hypoxic brain show widespread hyper-intensities, initially involving the basal ganglia, caudate, striatum and thalamus followed by the cortex and subcortical white matter, cerebellum and hippocampus. Conventional T1 and T2 weighted scans are usually normal and the presence of diffuse abnormal findings on DWI and FLAIR specifically correlate with a poor outcome. ${ }^{6}$ However MRI scan of brain of this patient revealed only mild involutional changes without any of the abnormalities mentioned above.

Prognostic factors for the recovery from the delirium following a brain injury include the duration of unconsciousness, hypoxia, hypoxaemia, and the extent of brain injury. ${ }^{3)}$ Age at the time of injury is less consistently implicated with some ${ }^{3-7)}$ but not all ${ }^{8)}$ studies observing association of poor prognosis with older age. This case illustrates the clinical manifestations and sequelae of pro- 
longed delirium following hypoxic ischaemic encephalopathy after cardiac arrest and highlights the importance of adopting a multidisciplinary management approach involving the coordinated care of medical and nursing teams to optimise management of patients suffering from such a crippling organic brain syndrome.

\section{REFERENCES}

1. Howard RS, Holmes PA, Koutroumanidis MA. Hypoxicischaemic brain injury. Pract Neurol 2011;11:4-18.

2. Groswasser Z, Cohen M, Costeff H. Rehabilitation outcome after anoxic brain damage. Arch Phys Med Rehabil 1989; 70:186-188.

3. Uguz F, Kayrak M, Cíçek E, Kayhan F, Ari H, Altunbas G. Delirium following acute myocardial infarction: incidence, clinical profiles, and predictors. Perspect Psychiatr Care 2010;46:135-142.

4. Haddad GG, Jiang C. O2 deprivation in the central nervous system: on mechanisms of neuronal response, differential sensitivity and injury. Prog Neurobiol 1993;40:277-318.

5. Eames P. Hysteria following brain injury. $J$ Neurol Neurosurg Psychiatry 1992;55:1046-1053.

6. Wijman CA, Mlynash M, Caulfield AF, Hsia AW, Eyngorn I, Bammer R, et al. Prognostic value of brain diffusionweighted imaging after cardiac arrest. Ann Neurol 2009;65: 394-402.

7. High WM Jr, Hall KM, Rosenthal M, Mann N, Zafonte R, Cifu DX, et al. Factors affecting hospital length of stay and charges following traumatic brain injury. J Head Trauma Rehabil 1996;11:85-96.

8. Schmidt JG, Drew-Cates J, Dombovy ML. Anoxic encephalopathy: outcome after inpatient rehabilitation. Neurorehabil Neural Repair 1997;11:189-195. 